\title{
Cardiac Tamponade Due to Inferior Vena Cava Filter Removal: A Case Report and Review of Literature
}

\author{
Sohaib Roomi ${ }^{1}$, Shujaul Haq ${ }^{1}$, Waqas Ullah ${ }^{1}$, Munnam S. Jafar ${ }^{2}$, Mahnoor Sherazi ${ }^{3}$ \\ 1. Internal Medicine, Abington Hospital - Jefferson Health, Abington, USA 2. Internal Medicine, Jinnah Hospital Lahore \\ (JHL)/Allama Iqbal Medical College (AIMC), Lahore, PAK 3. Internal Medicine, Fauji Foundation Hospital, Rawalpindi, \\ PAK \\ Corresponding author: Sohaib Roomi, roomigujjar251@gmail.com
}

\begin{abstract}
Cardiac tamponade is a condition characterized by the accumulation of pericardial fluid, compromising the hemodynamics of the circulation. It has several known causes, including traumatic injury to the pericardium, idiopathic, neoplastic or purulent pericarditis, and, rarely, iatrogenic etiology. Inferior vena cava (IVC) filter removal can lead to multiple complications including but not limited to IVC perforation, air embolism, pneumothorax or filter migration. Here, we present a case of a middle-aged woman presenting with cardiac tamponade after IVC filter removal. She was successfully managed with pericardiocentesis followed by pericardial window placement. As this case and literature review illustrates, cardiac tamponade is a rare but potentially devastating complication of IVC filter manipulation.
\end{abstract}

Categories: Cardiac/Thoracic/Vascular Surgery, Internal Medicine Keywords: inferior vena cava, cardiac tamponade

\section{Introduction}

Cardiac tamponade is a condition characterized by the pericardial fluid accumulating to compromise circulation hemodynamics. Cardiac tamponade can be caused by a number of different mechanisms including, but not limited to, mechanical trauma to the pericardium during chest wall injury, acute pericarditis, and myocardial rupture, especially after myocardial infarction or iatrogenic etiology. Inferior vena cava (IVC) filter manipulation can lead to IVC perforation, fracture or migration of IVC filter, air embolism, pneumothorax, insertion site thrombosis or wound infection. IVC filter manipulation leading to cardiac tamponade is very rare. Here, we present a case of a middle-aged woman presenting with cardiac tamponade after IVC filter removal.

Received 12/06/2019 Review began 01/13/2020 Review ended 02/11/2020 Published 02/14/2020

(c) Copyright 2020 Roomi et al. This is an open access article distributed under the terms of the Creative Commons Attribution License CC-BY 4.0., which permits unrestricted use, distribution, and reproduction in any medium, provided the original author and source are credited.

\section{Case Presentation}

A 49-year-old woman with a past medical history of pulmonary embolism presented for IVC filter removal, which was initially placed approximately three months prior to this hospitalization. She was not a smoker and had no history of chronic lung disease or occupational hazards. At that time, the patient had right leg deep vein thrombosis and right sub-segmental pulmonary artery embolism. Hematological workup was inconclusive and it was presumed to be provoked by prolonged bed rest in the setting of bariatric surgery. The patient had a history of intracranial aneurysm in the posterior circulation so long-term anticoagulation was not thought to be a safe option. She got IVC filter placed via the right femoral approach.

In the present hospitalization, interventional radiology (IR) guided IVC filter removal was done in the operating room (OR). Under X-ray fluoroscopy, a snare was inserted into the internal jugular vein, and the IVC filter was withdrawn from its hook (Figure 1). Per the OR notes, it was a difficult approach requiring manipulation of the snare. The patient was transferred after the procedure into an observation room. 


\section{Cureus}

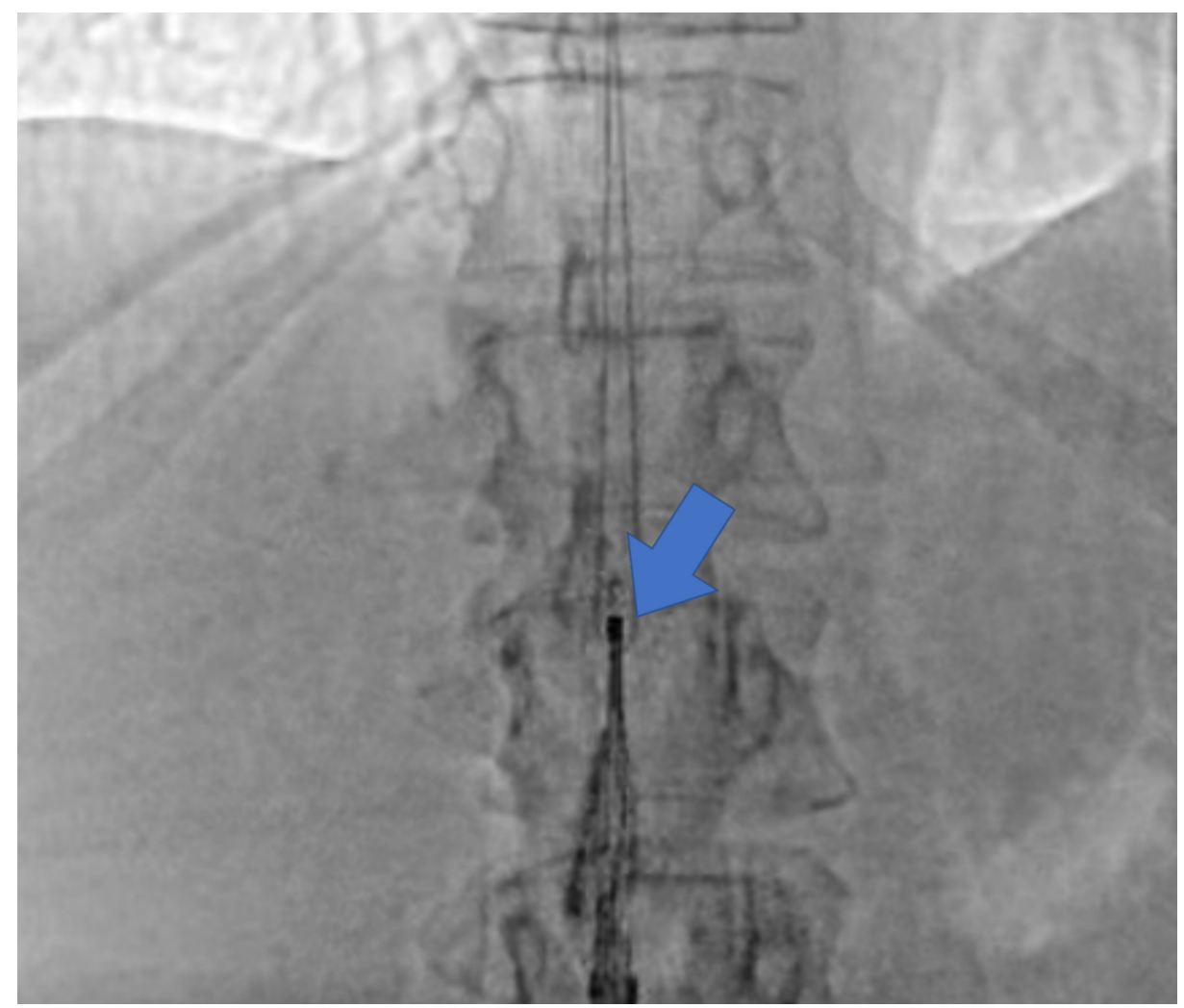

\section{FIGURE 1: Fluoroscopy revealing inferior vena cava filter removal via}

\section{snare}

Within an hour after the procedure, she developed pleuritic chest pain, hypotension, presyncope, and shortness of breath. She denied any other systemic symptoms like nausea, vomiting, diarrhea, joint pain, and bowel or bladder symptoms. Her physical examination showed a blood pressure of $85 / 60 \mathrm{mmHg}$, a pulse of 122 beats per minute, a respiratory rate of 26 breaths per minute, and an oxygen saturation of $92 \%$. She had a poor inspiratory effort. Her jugular venous pressure was elevated ( $9 \mathrm{~cm}$ of H2O). On cardiovascular examination, her heart sounds were markedly diminished, and the point of maximal impulse was nonpalpable. No murmurs, gallops, or rubs were appreciated. Her chest was clear on auscultation. The results of her abdominal and neurological examinations were also unremarkable. Her pertinent laboratory findings revealed PT/INR of 3.4, a potassium level of $3.1 \mathrm{mEq} / \mathrm{L}$, and hemoglobin of $10.6 \mathrm{~g} / \mathrm{dL}$. The other laboratory tests were unremarkable. Her chest X-ray revealed low lung volumes with bibasilar subsegmental atelectasis. An electrocardiogram (EKG) revealed sinus tachycardia, electrical alternans, low voltage QRS complexes, and a prolonged QT interval (Figure 2). 


\section{Cureus}

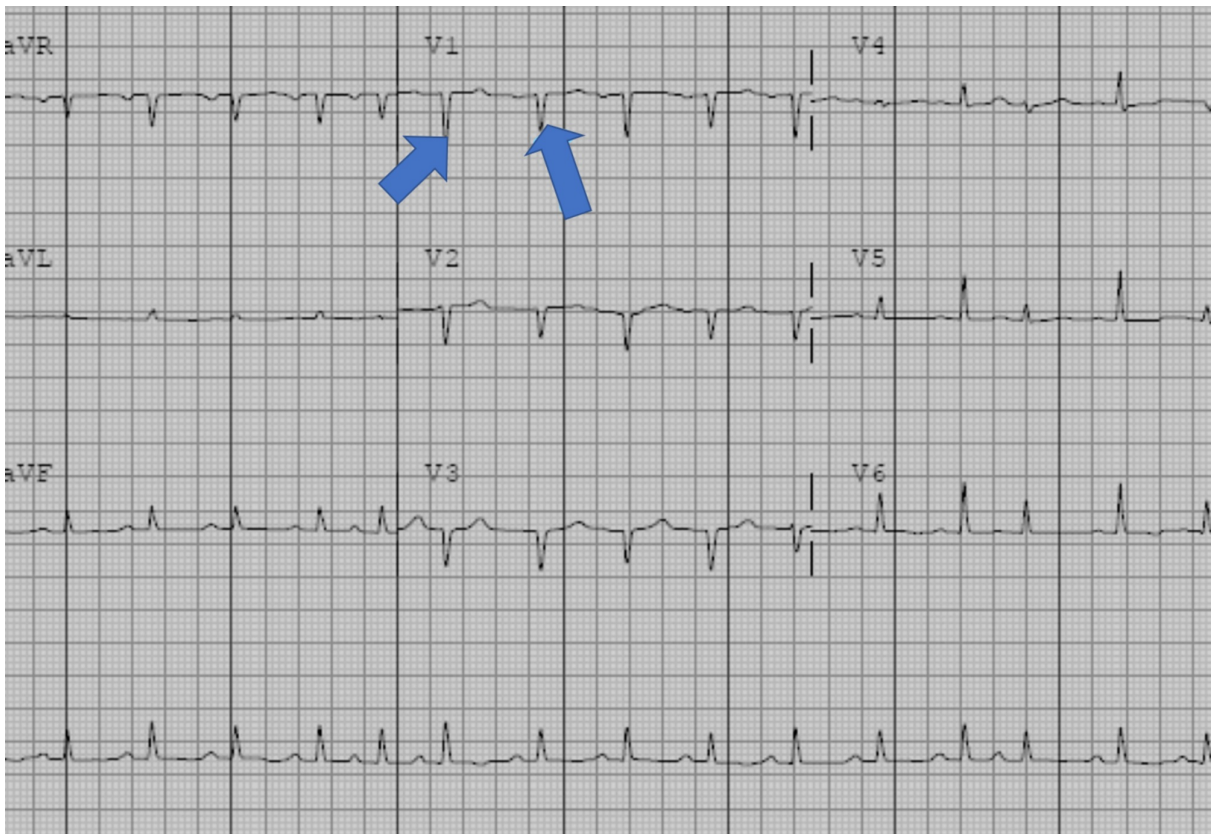

FIGURE 2: Electrocardiogram revealing sinus tachycardia, low voltage QRS complexes, and a prolonged QT interval

Variation in QRS amplitude with beats is called electrical alternans, a sign specific for cardiac tamponade. Arrows in the figure denote variation in QRS complex amplitude.

Bedside echocardiography (echo) at the symptom onset showed a large anterior, small to moderate lateral and posterior pericardial effusion. Left ventricular and right ventricular functions were grossly normal. Transthoracic echo revealed moderate to severe pericardial effusion, $2.1 \mathrm{~cm}$ in the largest dimension with right ventricular collapse during diastole consistent with the tamponade effect (Figure 3). It also showed a large echogenic mobile structure, consistent with pericardial thrombus.

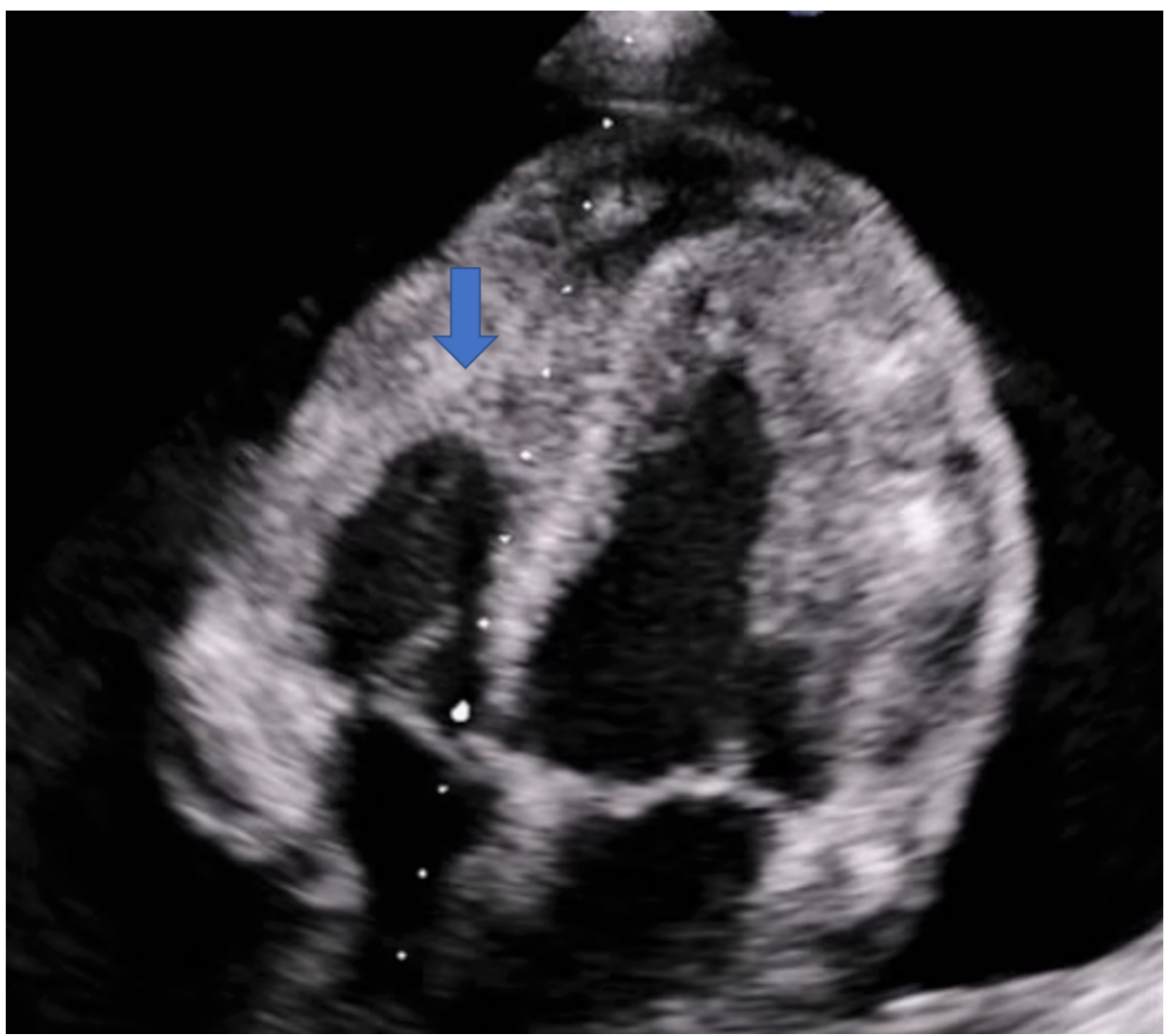


She was started on intravenous fluid resuscitation and immediately transferred to the OR. With an emergent pericardiocentesis, $300 \mathrm{~mL}$ of blood and a large clot was removed, and a pericardial window was placed. Postoperative transthoracic echocardiography (TTE) revealed that pericardial blood volume was significantly improved with only a small amount left anteriorly. The pericardial thrombus was no longer evident. She remained in the hospital for two days after the procedure. The drainage catheter was removed on the second day when it drained less than 25 ccs of fluid over the day. She was subsequently discharged in a stable condition, and an outpatient follow-up was advised.

\section{Discussion}

Cardiac tamponade occurs when pericardial fluid accumulates within pericardial space leading to reduced cardiac filling and, eventually, hypotension or shock. Cardiac tamponade has an estimated frequency of two per 10,000 and in $2 \%$ of chest gunshot wound victims [1]. Fluid accumulation within the pericardial space leads to a progressive decline in the cardiac filling. The resulting pericardial pressure forces the interventricular septum to bend towards the left side, which leads to a decrease in stroke volume [1]. We conducted a thorough search of the literature published to date with the search terms 'vena cava filter' and 'cardiac tamponade' on PubMed. We found a total of six published articles, which are summarized in Table 1 [2-7]. For this subset of patients, the average age is 48 years (range, 29 to 66 years) with a 5:1 ratio of male to female patients. The most common presentations were hypotension, tachycardia, and tachypnea in $66 \%$ of the population. About $16 \%$ of patients were asymptomatic, or there were not enough data mentioned. In $33 \%$ of the patients, chest pain and shortness of breath were the presenting concerns. Of these patients, $16 \%$ of patients had associated lower extremity weakness, and a similar percentage of patients had fatigue. Sixteen percent of the patients had an open skull fracture and multiple traumatic injuries incurred in a road traffic accident that caused them to seek immediate treatment leading to cardiac tamponade. History of deep venous thrombosis, pulmonary embolism, or both were present in $86 \%$ of the patient population. Deficiency of protein C, protein S or both were present in $33 \%$ of the patients. Other comorbidities included ulcerative colitis, hypertension, diabetes, and chronic kidney disease, each of them affecting $16 \%$ of the patient population. Cardiac tamponade in half of these patients was managed by pericardiocentesis followed by pericardial window placement and by cardiothoracic surgery in the other half. All patients recovered and were discharged in stable condition with $0 \%$ mortality. In five of these cases, cardiac tamponade occurred with IVC filter manipulation, and one case was associated with a filter in the superior vena cava. 


\section{Cureus}

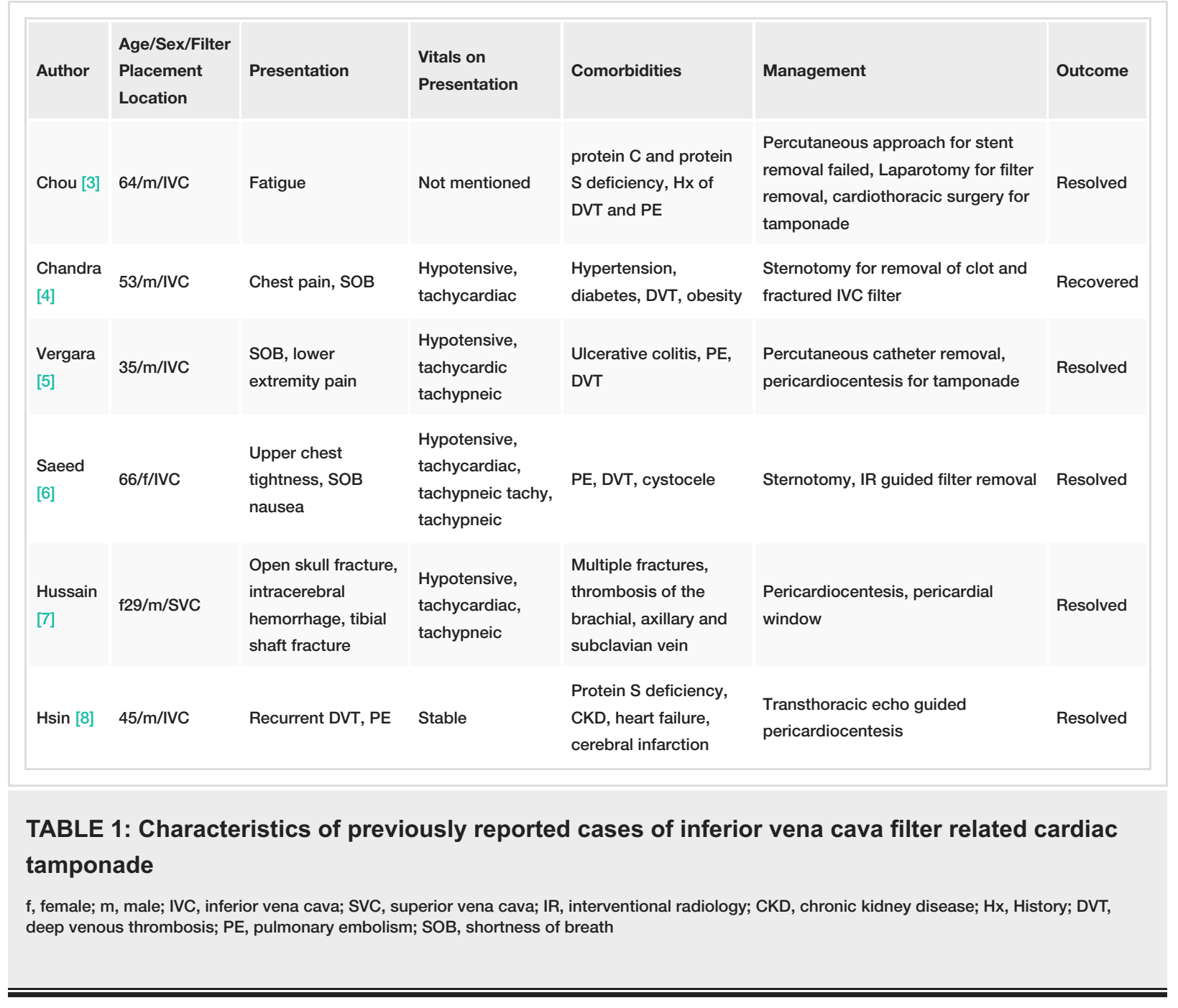

Our case marks only the seventh reported case of this rare complication associated with IVC filter removal or trauma. We believe more such cases if published, will provide awareness for the management of this rare entity. Cardiac tamponade can be classified as acute or subacute depending upon the timeline and acuity of presentation or necessity of immediate intervention. Acute tamponade generally occurs within minutes, often resembles cardiogenic shock, and requires urgent intervention [8]. It is caused by trauma, aortic rupture, or can be iatrogenic from the therapeutic or diagnostic procedure. Symptoms typically include shortness of breath, chest discomfort, lightheadedness, cough, and fatigue. The rest of the symptoms are caused by an underlying cause [9]. Subacute cardiac tamponade generally has delayed presentation over days to weeks. It may be caused by idiopathic, infectious, autoimmune, or neoplastic pericarditis. A small subset of patients may have regional cardiac tamponade when localized hematoma or loculated pericardial effusion compresses only selected heart chambers. Our case was an example of an acute cardiac tamponade where the patient developed symptoms of shortness of breath, chest discomfort, and lightheadedness within one hour of IR-guided IVC filter manipulation via the jugular vein. We believe that this might have occurred due to the manipulation of the vasculature and possible migration of small IVC fragments. There was no evidence of right atrial myocardial rupture to suggest the iatrogenic rupture of the myocardium. The diagnosis of cardiac tamponade is made by a combination of physical signs and imaging. Dilated neck veins, muffled heart sounds, and low arterial pressure constitute Beck's triad, which is present only in $10 \%$ to $40 \%$ of the patients having cardiac tamponade [10]. Imaging modalities most commonly employed include EKG, chest X-ray, and echo. EKG occasionally reveals sinus tachycardia, electrical alternans, and low-voltage QRS complex. Sinus tachycardia is more sensitive, but electrical alternans and low QRS voltage are specific for cardiac tamponade but rarely seen $[9,11]$. Findings on chest radiographs are relatively nonspecific and include enlarged cardiac silhouette with clear lung fields. At least $200 \mathrm{~mL}$ of fluid should accumulate in the pericardial space before it appears on the X-ray [9].

The echo is the diagnostic modality of choice. It shows the amount and location of pericardial fluid. In most patients with cardiac tamponade, echo reveals the diastolic collapse of the right atrium, right ventricle, or left heart collapse [12-13]. A prospective study on echo established sensitivity and specificity of $90 \%$ and $65 \%$, respectively, for cardiac tamponade detection in patients with large pericardial effusion [14]. Computed tomography and cardiovascular magnetic resonance imaging are usually not needed when echo is available. In our case, the patient's EKG showed sinus tachycardia and nonspecific T wave abnormalities. TEE revealed 
moderate to severe pericardial effusion, $2.1 \mathrm{~cm}$ in the largest dimension. It also showed a large echogenic mobile structure, consistent with pericardial thrombus, which is different from other cases included in the review. Management includes pericardiocentesis, cardiothoracic surgery, or conservative treatment depending upon the hemodynamic status of the patient [10]. In stable patients, careful monitoring of vital signs and serial echocardiograms is helpful. Fluid resuscitation can only be used to bridge the delay between pericardial fluid removal if the patient is unstable.

Pericardiocentesis involves catheter insertion under echo guidance at the best location, depending upon operator expertise, and left in place until fluid removal is less than $25 \mathrm{~mL} /$ day. Open surgical drainage, being more invasive, is performed under general anesthesia and preferred over pericardiocentesis when either pericardiectomy or pericardial biopsy is required or in cases where effusion is loculated. In a nonrandomized trial, percutaneous echo-guided pericardiocentesis resulted in lower complication and mortality rates compared to an open surgical approach [15]. After the procedure and initial stabilization, patients are observed with frequent vital monitoring, EKG, and echo to ensure adequate drainage of pericardial fluid. It is important to note that due to their hospitalization, these patients can still have a risk for venous thrombosis and pulmonary embolism. We believe that nonpharmacological management such as graduated compression stockings, intermittent pneumatic compression, and foot venous pump should be practiced in lieu of pharmacological management. In our patient, pericardiocentesis followed by the pericardial window was performed on both presentations, and a pericardial window was removed when less than 25 ccs of fluid was drained over 24 hours. Recurrence of pericardial effusion depends upon the etiology. The patient is usually discharged within 24 to 36 hours after stabilization and is advised to have a repeat echo as an outpatient to rule out any reaccumulation. In our patient, during the first admission, a pericardial catheter was kept in place for 30 hours and was removed when it drained less than $25 \mathrm{ccs} /$ day. She got readmitted with pericardial effusion, and pericardial catheter drained over $300 \mathrm{cc}$ fluid for 72 hours. Given that there are only a few published cases of cardiac tamponade resulting from IVC filter removal, there are no established guidelines for steps of management to date. More cases need to be reported so that data-driven guidelines can be established to manage this potentially devastating complication.

\section{Conclusions}

Cardiac tamponade is a rare but fatal complication in which the patient presents with tachycardia, shortness of breath, and visible venous pulsations in the neck. It should be suspected in all patients exhibiting these symptoms after insertion or removal of IVC filters. It is important to manage these complications on an emergency basis with a multidisciplinary approach involving surgical, anesthesia, and cardiovascular teams.

\section{Additional Information \\ Disclosures}

Human subjects: Consent was obtained by all participants in this study. Conflicts of interest: In compliance with the ICMJE uniform disclosure form, all authors declare the following: Payment/services info: All authors have declared that no financial support was received from any organization for the submitted work. Financial relationships: All authors have declared that they have no financial relationships at present or within the previous three years with any organizations that might have an interest in the submitted work. Other relationships: All authors have declared that there are no other relationships or activities that could appear to have influenced the submitted work.

\section{Acknowledgements}

I want to acknowledge the role of my Program Director Dr. Margot Boigon for providing us with the environment conducive to research and scholarly activities in addition to state of the art medical training.

\section{References}

1. Porth C: Essentials of Pathophysiology: Concepts of Altered Health States . Lippincott Williams \& Wilkins, Baltimore, MD; 2011.

2. Chou EL, Sgroi MD, Fujitani RM, Kabutey NK: Complex hybrid suprarenal inferior vena cava filter retrieval . Ann Vasc Surg. 2015, 29:125-19. 10.1016/j.avsg.2014.07.040

3. Chandra PA, Nwokolo C, Chuprun D, Chandra AB: Cardiac tamponade caused by fracture and migration of inferior vena cava filter. South Med J. 2008, 101:1163-4. 10.1097/SMJ.0b013e318172dd80

4. Vergara GR, Wallace WF, Bennett KR: Spontaneous migration of an inferior vena cava filter resulting in cardiac tamponade and percutaneous filter retrieval. Catheter Cardiovasc Interv. 2007, 69:300-2. 10.1002/ccd.21010

5. Saeed I, Garcia M, McNicholas K: Right ventricular migration of a recovery IVC filter's fractured wire with subsequent pericardial tamponade. Cardiovasc Intervent Radiol. 2006, 29:685-6. 10.1007/s00270-005-01367

6. Hussain SM, McLafferty RB, Schmittling ZC, et al.: Superior vena cava perforation and cardiac tamponade after filter placement in the superior vena cava: a case report. Vasc Endovascular Surg. 2005, 39:367-70. $10.1177 / 153857440503900412$

7. Hsin ST, Luk HN, Lin SM, Chan KH, Tsou MY, Lee TY: Detection of iatrogenic cardiac tamponade by transesophageal echocardiography during vena cava filter procedure. Can J Anaesth. 2000, 47:638-41. 


\section{Cureus}

10.1007/BF03018996

8. Spodick DH: Acute cardiac tamponade. N Engl J Med. 2003, 349:684. 10.1056/NEJMra022643

9. Reddy PS, Curtiss EI, Uretsky BF: Spectrum of hemodynamic changes in cardiac tamponade . Am J Cardiol. 1990, 66:1487. 10.1016/0002-9149(90)90540-h

10. Adler Y, Charron P, Imazio M, et al.: 2015 ESC Guidelines for the diagnosis and management of pericardial diseases: The Task Force for the Diagnosis and Management of Pericardial Diseases of the European Society of Cardiology (ESC) Endorsed by: The European Association for CardioThoracic Surgery (EACTS). Eur Heart J. 2015, 36:2921. 10.1093/eurheartj/ehv318

11. Bruch C, Schmermund A, Dagres N, et al.: Changes in QRS voltage in cardiac tamponade and pericardial effusion: reversibility after pericardiocentesis and after anti-inflammatory drug treatment. J Am Coll Cardiol. 2001, 38:219. 10.1016/s0735-1097(01)01313-4

12. Gillam LD, Guyer DE, Gibson TC, King ME, Marshall JE, Weyman AE: Hydrodynamic compression of the right atrium: a new echocardiographic sign of cardiac tamponade. Circulation. 1983, 68:294. 10.1161/01.cir.68.2.294

13. Kerber RE, Gascho JA, Litchfield R, Wolfson P, Ott D, Pandian NG: Hemodynamic effects of volume expansion and nitroprusside compared with pericardiocentesis in patients with acute cardiac tamponade. $\mathrm{N}$ Engl J Med. 1982, 307:929. 10.1056/NEJM198210073071506

14. Mercé J, Sagristà-Sauleda J, Permanyer-Miralda G, Evangelista A, Soler-Soler J: Correlation between clinical and Doppler echocardiographic findings in patients with moderate and large pericardial effusion: implications for the diagnosis of cardiac tamponade. Am Heart J. 1999, 138:759-64. 10.1016/s00028703(99)70193-6

15. Gumrukcuoglu HA, Odabasi D, Akdag S, Ekim H: Management of cardiac tamponade: a comparative study between echo-guided pericardiocentesis and surgery-a report of 100 patients. Cardiol Res Pract. 2011, 2011:197838. 10.4061/2011/197838 